\title{
Telomerase expression in intracranial tumours: prognostic potential for malignant gliomas and meningiomas
}

\author{
Maria Laura Falchetti, Roberto Pallini, Luigi M Larocca, Roberto Verna, \\ Ettore D'Ambrosio
}

\begin{abstract}
Aim-To evaluate the diagnostic value of telomerase expression in intracranial tumours.

Methods-98 surgical specimens from different neoplasms were analysed by the telomeric repeat amplification protocol (TRAP) and the presence of telomerase compared with the histological diagnosis and the proliferation index.

Results-A high degree of positivity for telomerase activity was found in glioblastomas and atypical/anaplastic meningiomas. Telomerase activity was poorly detected in anaplastic astrocytomas.

Conclusions-The TRAP assay seems to be a valuable index for identifying meningeal tumours with aggressive behaviour. (f Clin Pathol 1999;52:234-236)
\end{abstract}

Istituto di Medicina

Sperimentale, CNR,

Viale Marx, 43. 00137

Rome, Italy

M L Falchetti

E D'Ambrosio

Istituto di

Neurochirurgia,

Università Cattolica,

Rome, Italy

R Pallini

Istituto di Anatomia Patologica, Università Cattolica, Rome, Italy

L M Larocca

Dipartimento di

Medicina

Sperimentale,

Università "La

Sapienza", Rome, Italy

R Verna

Correspondence to:

Dr D'Ambrosio.

email:

ettore@biocell.irmkant.rm.cnr.

Accepted for publication

19 October 1998

Keywords: intracranial tumours; telomerase; TRAP

The term telomere refers to the extreme ends of eukaryotic chromosomes which are composed of tandem repeated short sequences associated with specific proteins. These structures protect the chromosomes from degradation and prevent them from fusing with each other and from recombining with internal DNA. Owing to the incapacity of DNA polymerase to replicate the very end of linear chromosomes, ${ }^{1}$ telomeres shorten at each cell division in somatic cells. In contrast, in germ line cells, telomere length is maintained by the activity of telomerase, a ribonucleoprotein complex which adds the human telomeric TTAGGG repeat to the chromosome ends. The enzymatic activity of telomerase is strictly regulated. In human somatic cells of different lineages, it is not detectable and telomere length decreases with increasing number of cell divisions in vitro and with age in vivo. Reactivation of telomerase seems to be related to unrestrained cell proliferation and cancer progression. ${ }^{2}$ Mutations may occur that reacti-

Table 1 Clinical profile of patients ( $n=98$ ) undergoing neurosurgery for resection of intracranial tumours

\begin{tabular}{llll}
\hline & Neuroepithelial tumours & Meningeal tumours & Miscellaneous tumours \\
\hline $\mathrm{n}$ & 51 & 31 & 16 \\
Age, years (range) & $59.3(8$ to 78$)$ & $55.9(41$ to 69$)$ & 46.1 (13 to 82$)$ \\
Sex $(\mathrm{M} / \mathrm{F})$ & $32 / 19$ & $13 / 18$ & $9 / 7$ \\
Duration of symptoms, & & & \\
$\quad$ months (range) & $9.1(1$ to 36$)$ & $25.2(6$ to 60$)$ & $28.6(3$ to 60$)$ \\
Follow up, months (range) & $8.0(3$ to 24$)$ & $11.4(6$ to 19$)$ & $8.6(4$ to 24$)$ \\
Survival rate, \%^ & 58.8 & 93.5 & 81.2
\end{tabular}

*The survival rate was calculated at the end of the follow up period. vate telomerase and stabilise telomere length by the addition of telomeric repeats, allowing unlimited proliferation. Using the highly sensitive telomeric repeat amplification protocol (TRAP) method, ${ }^{2}$ various human cancers have been analysed and a good correlation shown between telomerase activity and tumour malignancy. ${ }^{3-6}$ In some cases, telomerase activity has also given valuable information on prognosis; for example, high levels of telomerase are associated with a poor outcome in neuroblastomas and gastric cancer. ${ }^{4}$ In order to evaluate the diagnostic value of the TRAP assay and to gain a better understanding of the role of telomerase in cell immortalisation, we analysed a series of intracranial tumours and compared the results of the TRAP assay with histological diagnosis and proliferative index.

\section{Methods}

Tissue specimens were obtained from 98 patients who underwent surgery for resection of intracranial tumours. Clinical features of patients are summarised in table 1. No procedures were performed for research purposes only. The tumours were classified for histology according to the World Health Organisation criteria. ${ }^{7}$ The proliferative index was calculated as the percentage of tumour cell nuclei immunoreactive for $\mathrm{Ki} 67$ to total tumour nuclei. In each specimen we studied at least 500 neoplastic cells, as defined by histological criteria. For telomerase analysis, the surgical specimens were immediately frozen in liquid nitrogen and stored at $-80^{\circ} \mathrm{C}$ until use. Protein extracts were prepared using CHAPS containing extraction buffer as described by Hiyama et $a l,{ }^{4}$ and protein concentration was determined with BCA protein assay reagent (Pierce). An amount of $2 \mu \mathrm{g}$ of protein was used for telomerase assays. The samples were analysed using a modified TRAP test. ${ }^{8}$ Briefly, we redesigned the downstream primer CX to minimise the possibility of primer dimer interaction, resulting in improved sensitivity and reproducibility of the test as compared with the original TRAP protocol.

\section{Results}

Results are shown in table 2. Our series consisted mainly of astrocytic tumours (45 cases) and meningeal tumours (31 cases). There were 11 recurrent tumours including three glioblastomas, one anaplastic astrocytoma, five anaplastic/atypical meningiomas, one haemangioblastoma, and one epidermoid 
Table 2 Telomerase expression in intracranial tumours

\begin{tabular}{|c|c|c|c|c|}
\hline \multirow[b]{2}{*}{ Histology (WHO grade) } & \multirow[b]{2}{*}{$n$} & \multicolumn{2}{|c|}{ Telomerase activity } & \multirow{2}{*}{$\begin{array}{l}\text { Proliferative } \\
\text { index, mean } \\
\text { (min-max) }\end{array}$} \\
\hline & & $\begin{array}{l}\text { Positive } \\
(\%)\end{array}$ & $\begin{array}{l}\text { Negative } \\
(\%)\end{array}$ & \\
\hline \multicolumn{5}{|c|}{ Neuroepithelial and meningeal tumours } \\
\hline Astrocytic tumours & 45 & & & \\
\hline Astrocytoma (II) & 4 & $0(0)$ & $4(100)$ & $2.2(1$ to 3$)$ \\
\hline Anaplastic astrocytoma (III) & 11 & $3(27.3)$ & $8(72.7)$ & $23.2(3$ to 45$)$ \\
\hline Glioblastoma (IV) & 30 & $25(83.3)$ & $5(16.6)$ & $19.8(5$ to 40$)$ \\
\hline Oligodendrogliomas & 3 & & & \\
\hline Oligodendroglioma (II) & 2 & 1 & 1 & $3(2$ to 4$)$ \\
\hline Oligo-astrocytoma (II) & 1 & 1 & 0 & 7 \\
\hline Other neuroepithelial tumours & 3 & & & \\
\hline Choroid plexus papilloma (I) & 1 & 1 & 0 & 1 \\
\hline Pineoblasoma (IV) & 1 & 1 & 0 & 50 \\
\hline Neuroblastoma (IV) & 1 & 0 & 1 & 12 \\
\hline Meningeal tumours & 31 & & & \\
\hline Meningioma (I) & 21 & $0(0)$ & $21(100)$ & $0.9(0.5$ to 2$)$ \\
\hline Atypical meningioma (II) & 8 & $6(75)$ & $2(25)$ & $14.2(5$ to 43$)$ \\
\hline Anaplastic meningioma (III) & 2 & $2(100)$ & $0(0)$ & $65(60$ to 70$)$ \\
\hline \multicolumn{5}{|l|}{ Other tumours } \\
\hline Miscellaneous tumours & 16 & & & \\
\hline Chondrosarcoma & 1 & 0 & 1 & 60 \\
\hline Craniopharyngioma & 2 & 0 & 2 & $16(14$ to 18$)$ \\
\hline Epidermoid cyst & 1 & 0 & 1 & 0 \\
\hline Pituitary adenoma & 8 & $2(25)$ & $6(75)$ & $2.5(1$ to 4$)$ \\
\hline Cavernous angioma & 2 & 0 & 2 & 0 \\
\hline Haemangioblastoma & 1 & 0 & 1 & 0 \\
\hline Primary brain lymphoma & 1 & 1 & 0 & 70 \\
\hline
\end{tabular}

${ }^{\star}$ Diffuse large B cell lymphoma according to the REAC classification. ${ }^{9}$

cyst. Two of the recurrent glioblastomas had a previous diagnosis of anaplastic astrocytoma. In the other recurrent tumours, histology was unchanged at reoperation.

Overall, telomerase activity was detected in 43 cases $(43.9 \%)$. The highest degree of telomerase activity was found in glioblastomas and atypical/anaplastic meningiomas (fig 1). It seems that astrocytic tumours show increasing percentages of telomerase positivity with tumour progression toward malignancy. A similar correlation was observed in meningeal

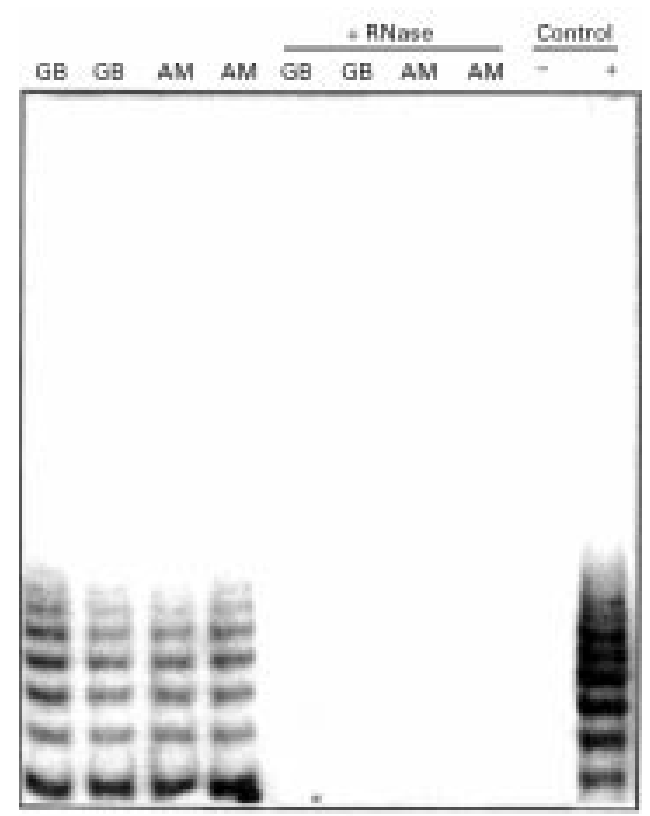

Figure 1 Telomerase activity in malignant intracranial tumours. Telomerase activity of glioblastoma ( $G B$, lanes 1 and 2) and anaplastic meningioma (AM, lanes 3 and 4). Lanes 5-8 show TRAP reactions performed on RNAse pretreated extracts of samples 1-4, respectively. In lane 9, the reaction was performed with no extract (negative control) and lane 10 shows telomerase activity of the human cell line U937 (positive control). tumours. Telomerase activity was detected in 25 of 30 glioblastomas $(83.3 \%)$. By contrast, only three of 11 anaplastic astrocytomas $(27.3 \%)$ had a positive telomerase reaction. This discrepancy in the presence of telomerase was not associated with a marked difference in the proliferative index, which averaged $19.8 \%$ and $23.2 \%$ in the glioblastomas and anaplastic astrocytomas, respectively. In addition, we did not find a significant difference in the proliferative index between glioblastomas with a positive telomerase reaction and those with a negative reaction ( $\mathrm{p}>0.05$, Student $t$ test).

On the basis of the clinical data and histology from previous surgery, three glioblastoma cases could be considered secondary tumours. Two of them showed a positive telomerase reaction, while the third was negative. The presence of polymerase chain reaction or Taq inhibitors in negative TRAP reactions seems unlikely because negative extracts retested in the presence of an internal DNA standard remained negative, while the internal DNA standard was easily amplified (data not shown).

\section{Discussion}

The different telomerase pattern between glioblastomas and anaplastic astrocytomas, which has also been reported by others, ${ }^{6}$ is intriguing. Most of glioblastomas are thought to arise by progression of anaplasia from pre-existing astrocytomas. Because of the heterogeneous nature of anaplastic astrocytomas it is possible that the astrocytoma samples used for telomerase analysis, though including a proliferating cell population as demonstrated by the $\mathrm{Ki} 67$ labelling index, missed the anaplastic foci. Alternatively, it is possible that in anaplastic astrocytomas the mechanisms of DNA replication do not involve telomerase, a conclusion that would favour the concept that glioblastomas represent a primary cancer of the brain. In meningeal tumours, all the WHO grade I meningiomas were found to be negative for telomerase activity, whereas six of eight atypical meningiomas (WHO grade II) and both cases of anaplastic meningioma (WHO grade III) showed telomerase activity (table 2 and fig 1). Although the small number of atypical/anaplastic meningiomas does not allow definitive conclusions to be drawn, the present work and a similar study ${ }^{10}$ indicate that telomerase detection may help in differentiating benign meningiomas from meningiomas with aggressive biological behaviour, where conventional histology may not be conclusive.

The other tumours from our study (table 2) formed a heterogeneous group of neoplasms and only limited conclusions can be drawn. The overall picture emerging from this miscellaneous group of intracranial tumours is that telomerase cannot be considered a specific feature of tumour malignancy, since it was detected both in highly malignant tumours, such as a pineoblastoma and a lymphoma, and also in benign tumours, such as pituitary adenomas and a choroid plexus papilloma. Conversely, telomerase was lacking in a malignant neuroblastoma and a chondrosarcoma. It has been suggested that in some neuroblasto- 
mas which do not show telomerase activity, the telomeres might eventually reach a critically shortened length, with the tumour undergoing spontaneous remission. ${ }^{4}$

In conclusion, our study shows that the assessment of telomerase activity may influence tumour prognosis, and possibly treatment, in malignant gliomas and meningiomas. Telomerase analysis may also be a valuable contribution in the differential diagnosis of glioblastoma and anaplastic astrocytoma, and in the detection of the atypical meningiomas. The large number of telomerase negative intracranial tumours implies that alternative mechanisms to stabilise telomeres may be present. Importantly, the discordance observed between telomerase activity and proliferation index suggests that telomerase may be a novel marker for brain cancer.
1 Watson JD. Origin of concatameric T7 DNA. Nature New Biol 1972;239:197-201.

2 Kim NW, Piatyszek MA, Prowse K, et al. Specific association of human telomerase activity with immortal cells and cancer. Science 1994;266:2011-15.

3 Avilion AA, Piatyszek MA, Gupta J, et al. Human telomerase RNA and telomerase activity in immortal cell lines and tumor tissues. Cancer Res 1996;56:645-50.

4 Hiyama E, Hiyama K, Yokoyama $\mathrm{T}$, et al. Correlating telomerase activity levels with human neuroblastoma telomerase activity levels with human

5 Hiyama E, Yokoyama T, Tatsumoto N, et al. Telomerase activity in gastric cancer. Cancer Res 1995;55:3258-62.

6 Langford LA, Piatyszek MA, Xu R, et al. Telomerase activity in human brain tumors. Lancet 1995;346:1267-8.

7 Kleihues P, Burger PC, Scheithauer BW. The new WHO classification of brain tumors. Brain Pathol 1993;3:255-6

8 Falchetti ML, Levi A, Molinari P, et al. Increased sensitivity and reproducibility of TRAP assay by avoiding direct and reproducibility of TRAP assay by avoiding
primers interaction. Nucleic Acids Res 1998;26:862-3.

9 Harris NL, Jaffem ES, Stain H, et al. A revised European-American classification of lymphoid neoplasms: a proposal from the international lymphoma study group. Blood 1994;84:1361-92.

10 Langford LA, Piatyszek MA, Xu R, et al. Telomerase activity in ordinary meningiomas predicts poor outcome. Hum Pathol 1997;28:416-20. 